\title{
FragmentaÇÃo FlORESTAL: BREVES CONSIDERAÇões TEÓRICAS SOBRE EFEITOS DE BORDA ${ }^{1}$ \\ Pablo José Francisco Pena Rodrigues ${ }^{2,3}$ \& Marcelo Trindade Nascimento ${ }^{2}$
}

\begin{abstract}
RESUMO
(Fragmentação florestal: breves considerações teóricas sobre efeitos de borda) Os processos de fragmentação florestal atualmente representamum dos principais riscos à biodiversidade global. Neste contexto é muito importante considerar estas transformações artificiais aos ecossistemas para compreender até mesmo padrões e processos ecológicos naturais. Neste trabalho é feita uma sinopse teórica que inclui o desenvolvimento de alguns conceitos e uma análise crítica destes, mesmo na falta de um arcabouço conceitual unificador sobre fragmentação florestal. São discutidas algumas teorias sobre limites (i.e. bordas) artificiais e naturais tendo como base as transformações ao longo do tempo e fenômenos de retração e expansão de comunidades e ecossistemas. A sucessão ecológica é brevemente discutida com base nas visões conflitantes de Clements e Gleason e mencionamos a importância de rever alguns modelos sucessionais para elucidar determinados aspectos dos efeitos de borda. Sobre estes efeitos é esboçada uma breve perspectiva histórica da evolução de alguns conceitos. Embora exista um conhecimento relativamente vasto sobre os efeitos de borda afirmamos que ainda é muito difícil prever a trajetória dos processos ecológicos em bordas assim como as transformações nos padrões naturais.
\end{abstract}

Palavras-chave: desmatamento, extinção, sucessão, teoria ecológica, limites.

\section{Abstract}

(Forest fragmentation: brief theoretical considerations about edge effects) Forest fragmentation is one of the main threats to global biodiversity. Considering artificial changes in ecosystems is essential to understand even natural ecological patterns and processes. A theory overview is performed, including the improvement of concepts and critical analysis, despite the lack of a unifying conceptual framework about forest fragmentation. We also discuss some theories about natural and artificial limits (i.e. boundaries) based on transformations over time and communities/ecosystems retraction and expansion. Ecological succession is briefly discussed using some aspects of Clement's and Gleason's conflicting viewpoints and pointing out the need for reviewing some succession models in order to understand some edge effects. About these effects we draft a concise historical perspective regarding the evolution of some concepts. Despite the relatively wide knowledge on edge effects we argue that it is very difficult to predict ecological processes pathways on edges as well as changes on natural patterns.

Key-words: deforestation, extinction, succession, ecological theory, limits.

\section{A fragmentaÇão}

Historicamente, a cobertura florestal do planeta tem sido reduzida através do desmatamento, principalmente para a expansão de fronteiras agrícolas (Tilman 1999). Com isto, florestas anteriormente contínuas encontramse agora dispostas em fragmentos remanescentes freqüentemente definidos pelos seus limites físicos (e.g. bordas, sensu Forman 1995). Dentre estas florestas, a Mata Atlântica, um dos principais centros de biodiversidade do mundo (WCMC 1992) detém apenas cerca de $7 \%$ de sua cobertura original (Myers et al. 2000; Morellato \& Haddad 2000) constituindo certamente um dos biomas mais atingidos por este crescente desmatamento. O impacto causado tem como conseqüências, além da disposição dos remanescentes florestais em fragmentos, a extinção de hábitats e espécies (Saunders et al. 1991; Tilman et al. 1994; Laurance \& Bierregaard 1997). Os processos envolvidos e suas consequiências têm sido

\footnotetext{
Artigo recebido em 03/2005. Aceito para publicação em 12/2005.

${ }^{1}$ Parte da tese do primeiro autor desenvolvida no Curso de Doutorado em Biociências e Biotecnologia da Universidade Estadual do Norte Fluminense (fevereiro de 2004)

${ }^{2}$ Universidade Estadual do Norte Fluminense. Laboratório de Ciências Ambientais.

${ }^{3}$ Instituto de Pesquisas Jardim Botânico do Rio de Janeiro. Rua Pacheco Leão 915, Botânica Sistemática sala 210, CEP 22460-030, Rio de Janeiro - RJ.

Apoio Financeiro: Fundação Estadual do Norte Fluminense/UENF

Jardim Botânico do Rio de Janeiro/PETROBRAS/Convênio 610.4.025.02.3
} 
comumente chamados de "fragmentação florestal".

Utilizando o conhecimento atual, são observados determinados padrões e processos relativamente consistentes (Laurance \& Yensen 1991; Murcia 1995; Laurance \& Bierregaard 1997), porém muitos permanecem relativamente frágeis (Pimm 1998; Harrison \& Bruna 1999; Debinski \& Holt 2000; Laurance et al. 2002). Parte deste problema reside na difícil interpretação dos fenômenos biológicos relativos à fragmentação com base em um corpo teórico aparentemente limitado acerca das florestas tropicais onde determinados conceitos e teorias são ainda hipóteses. Dentre os vários exemplos pode ser destacado o importante conceito de comunidade, o qual muitas vezes é vago e de difícil aplicação (Shrader-Frechette \& McCoy 1993). Assim como este, as discussões acerca da distinção entre fragmentação e perda de habitat são igualmente confusas. Enquanto alguns autores consideram a fragmentação apenas uma progressiva subdivisão do habitat (e.g. Fahrig 1997), sem vinculá-la diretamente a perda de habitat, outros utilizam o termo de forma genérica tanto indicando perda ou fragmentação de habitats (e.g. Holt et al. 1995). Contudo, aparentemente, há consenso de que a perda de habitat e a fragmentação em geral ocorrem simultaneamente modificando a biota (Kareiva \& Wennergren 1995; Fahrig 2003). Tais concepções esbarram mais uma vez em falhas conceituais onde os fragmentos remanescentes são interpretados como elementos geométricos (com limites definidos) em uma paisagem. Esta complexa paisagem em transformação já sinaliza que os limites, ainda que aparentemente sejam abruptos, dependem em grande parte dos fenômenos que ocorrem na matriz (Pimm 1998; Mesquita et al. 1999). Estes por sua vez, juntamente com as características do remanescente, irão determinar a intensidade dos efeitos de borda locais. Neste sentido, vários estudos têm sido desenvolvidos para tentar elucidar os mecanismos e efeitos ecológicos da fragmentação (e.g. Laurance \& Bierregaard 1997; Debinski \& Holt 2000).
Acredita-se que as escalas de espaço e tempo das transformações impostas pela fragmentação artificial em termos relativos são temporalmente muito curtas e espacialmente largas quando comparadas a processos naturais de fragmentação. Na evolução das florestas tropicais há fortes indícios de que existiram períodos de descontinuidade (vide Cox \& Moore 1994), os quais podem ser interpretados como eventos de fragmentação natural. Tais períodos estariam associados às flutuações climáticas que determinariam processos de expansão e retração dos hábitats, ecossistemas e biomas. Em períodos mais secos as florestas tropicais das Américas e da África estariam mais restritas em extensão, apresentando-se como ilhas em um mar de florestas sazonais (mais secas). Atualmente as florestas estariam experimentando o máximo de expansão, o qual teria sido alcançado durante o período Quaternário (Whitmore1991).

Em contrapartida, os níveis de fragmentação observados atualmente, conforme já mencionado, têm reduzido em grande escala as florestas tropicais, possivelmente ocasionando mais um grande evento de extinção em massa. Contudo, se as atuais taxas de desmatamento não produzirem uma eliminação completa de tais ecossistemas, talvez seja possível reverter este processo de degradação. Nesta perspectiva, o fato de que as florestas tropicais em sua evolução de certa forma sobreviveram a períodos de descontinuidade, talvez indique de que é possível haver mecanismos de proteção contra fenômenos relacionados à fragmentação que operam em distintos níveis hierárquicos. Ainda que seja difícil definir claramente "continuidade", principalmente em ecossistemas intrinsecamente heterogêneos (vide MacIntosh 1991) tidos como mosaicos heterogeneamente estruturados (sensu Watt 1947), perceber determinados limites tem sido bastante útil. Neste sentido, uma das interpretações advindas da teoria ecológica assume que tais limites podem ser graduais ou abruptos (vide Begon et al. 1995), dependendo do ecossistema ou comunidade. Esta concepção aparentemente 
constitui um dos pontos de convergência entre a perspectiva de super-organismo altamente integrado, desenvolvida inicialmente por Clements (1916 apud Begon et al. 1995), e a visão reducionista de Gleason (1927) onde as comunidades constituiriam assembléias não coesivas nas quais os fenômenos sobre os indivíduos assumiriam grande importância. Vários trabalhos têm sido desenvolvidos utilizando tais idéias e mesmo assim ainda não há consenso acerca dos processos e padrões relativos a tais áreas limítrofes (Laurance et al. 2001). Este fato remete novamente à idéia de limites artificiais (bordas) os quais podem ser semelhantes, em alguns aspectos, aos limites naturais.

Os danos causados pela fragmentação artificial, e conseqüente proliferação de bordas, aparentemente são extensos influenciando praticamente todo o ecossistema e as respectivas comunidades (Laurance 2000). Ou seja, há indícios de que tais impactos não se restringem à borda e atingem também porções teoricamente mais protegidas, distantes do limite artificial. Contudo, neste novo limite o ecossistema e as comunidades em geral estão expostos a uma série de condições distintas daquelas experimentadas anteriormente (Matlack 1993; Kapos et al. 1997). Estas condições apresentam inúmeras variações, que em grande parte se relacionam aos variados tipos de atividades humanas realizadas onde houve o desmatamento. Como conseqüência direta ou indireta são frequentemente observados vários efeitos nocivos à floresta $\mathrm{e}$ dentre estes, destacam-se os efeitos de borda (Lovejoy et al. 1986; Saunders et al. 1991; Laurance \& Bierregaard 1997). Alguns estudos sugerem que tais efeitos são semelhantes ao que ocorre em clareiras naturais (sensu Denslow 1987). Em termos gerais é possível sugerir que uma grande clareira pode apresentar um limite florestal abrupto em parte similar ao observado em uma borda criada artificialmente. Contudo, considerando válida tal analogia, há consenso de que a intensidade de determinados fenômenos será maior nas bordas artificiais.
Isto devido à escala espacial de criação dos amplos limites artificiais (bordas) e do fato de que estes foram originados a partir de processos exógenos, induzidos pelo homem. Ainda que as causas dos processos de formações de clareiras naturais em geral possam ser interpretadas como exógenas (Brokaw 1982) uma vez que freqüentemente advém de tempestades e fortes ventos, em sua essência ainda são distintas do resultante de atividades humanas. Além disto, como já citado, os locais adjacentes aos remanescentes florestais podem apresentar atividades que atingem a borda propriamente dita (e.g. queimadas, exposição a produtos químicos) ou trechos mais interiores (e.g. caça, extração de madeira, chuva ácida). Há inclusive determinados efeitos de borda que podem penetrar remanescentes florestais por mais de 400 metros ou até quilômetros (Laurance 2000). Neste caso, o fogo, em algumas regiões, tem sido relatado como um impacto recorrente e altamente invasivo (Cochrane \& Schulze 1999; Cochrane 2001).

\section{OS LIMITES}

"Boundaries exist as discontinuities
between contrasting habitats, and may
be expressed as ecótones, gradients, or
edges." (Pickett et al. 2000)

Os limites entre os ecossistemas são estudados praticamente desde o início da ecologia. Dentre os diversos autores que trataram deste tema são freqüentemente destacados Clements e Gleason que, mesmo com suas distintas visões acerca dos ecossistemas, mencionadas anteriormente, apontaram para o papel fundamental destas áreas limítrofes. Neste sentido eram reconhecidos ambientes de transição ("ecótones") que poderiam ser graduais ou abruptos conforme já citado. A importância destes ambientes é atribuída em grande parte à manutenção da integridade dos ecossistemas e comunidades, considerando inclusive a manutenção da área ocupada por estes assim como os processos e padrões envolvidos (Begon et al. 1995). 
A referida manutenção opera em geral em uma escala de tempo relativamente curta. Ou seja, os ecossistemas e as comunidades estão em constante transformação. Logo, até mesmo seus limites se modificam ao longo do tempo. Assim sendo, o ponto de equilíbrio onde haveria uma estabilização dos fluxos de energia, por exemplo, é também dependente de escala (vide Pimm 1991). No longo prazo, o acúmulo de entropia sempre tenderia a desequilibrar os ecossistemas (Holling 1973), o que poderia ocasionar mudanças em suas escalas espaciais. Tais idéias já foram anteriormente exploradas e até certo ponto é consenso de que devam existir inúmeros pontos de equilíbrio ao longo da evolução dos ecossistemas. Isto remete novamente a natureza dinâmica dos ecossistemas e a já referida alteração da escala espacial com fenômenos de expansão e retração.

Portanto os limites sejam estes abruptos ou graduais, podem representar a primeira frente de transformação. As mudanças verificadas nestes poderiam indicar uma tendência de retração ou expansão do referido ecossistema. Neste sentido, as modificações nos trechos imediatamente adjacentes a estes limites poderiam até mesmo catalisar mudanças em todo o ecossistema e nas respectivas comunidades (Laurance \& Yensen 1991). Estas mudanças comprometeriam inclusive, seus processos evolutivos que, em última análise, determinam sua permanência no tempo.

Atualmente são observadas transformações que resultam principalmente na extinção de espécies e comunidades (Saunders et al. 1991; Tilman et al. 1994; Laurance \& Bierregaard 1997). Embora tal degradação seja extensa, não é possível determinar se este processo irá promover uma extinção em massa que comprometa toda a vida no planeta. Neste aspecto, a despeito de todas as ondas de extinções experimentadas e de sua possível periodicidade (Patterson \& Smith 1989; Moses 1989), a biota parece algo extremamente persistente. Desta forma, os ecossistemas e as comunidades provavelmente continuarão a existir, e de forma análoga, as espécies também irão evoluir neste novo cenário de fragmentação e degradação intensa. Entretanto, há consenso de que a redução crescente na escala espacial decorrente da expansão das atividades humanas, irá promover um empobrecimento dos ecossistemas naturais, sobretudo das florestas tropicais (Whitmore 1991; Myers 1994).

O complexo cenário atual torna difícil prever as conseqüências destas transformações, incluindo-se neste caso os fenômenos relacionados à fragmentação. Contudo, há indícios de que os limites artificiais diferem dos limites supostamente naturais. Mesmo considerando algumas semelhanças, os limites antropogênicos em sua essência configuram uma nova situação, conforme já salientado. O caminho evolutivo que tais transformações irão impor ainda é desconhecido. Porém, as transformações mencionadas também sugerem que talvez seja útil reinterpretar as idéias acerca dos limites.

\section{A SUCESSÃo ECOLÓGiCa E OS LIMITES ARTIF ICIAIS}

As transformações das comunidades vegetais que compõem os ecossistemas em determinadas escalas de espaço e tempo são freqüentemente interpretadas utilizando conceitos relativos à sucessão ecológica (vide McCook 1994). O conjunto de idéias acerca da sucessão foi inaugurado, em moldes contemporâneos, por Thoreau (1860), e revela um padrão ao qual grande parte dos processos de sucessão vegetal aparentemente está subordinada. Os processos de sucessão vegetal devem, teoricamente, ser iniciados por espécies pioneiras (sensu Budowski 1965; Whitmore 1989), as quais apresentariam, em geral, determinados atributos tais como tolerância à alta insolação e a escassez de nutrientes. Na medida em que avançam tais processos ocorreriam modificações do ambiente que permitiriam a entrada das espécies mais tardias (shade tolerants). As espécies pioneiras neste caso poderiam também atuar como facilitadoras para a entrada de 
espécies características de estágios sucessionais mais avançados. No momento seguinte seria esperado que as espécies mais tardias excluíssem por competição as espécies típicas de estágios sucessionais menos avançados. Neste sentido, os processos de sucessão ecológica em florestas tenderiam a um estado mais equilibrado, mais complexo, mais estável, denominado clímax (vide Whitmore 1991).

Tais idéias foram inicialmente sumarizadas por Clements (1916 apud Begon et al. 1995) em sua concepção organísmica das comunidades e ecossistemas. A "hipótese de monoclimax", onde somente distúrbios fariam retroceder os processos sucessionais e o "revezamento florístico" no qual haveria um sistema ordenado e hierárquico de trocas na comunidade, constituem o cerne do pensamento holístico e organísmico (vide Odum 1964). Em tal analogia é freqüente a afirmativa de que a sucessão inclui a "ontogenia" e a "filogenia" das formações clímax. Por outro lado, as concepções adotadas por Cowles (1926) e Gleason (1927), em grande parte se contrapõem a esta visão. Neste sentido, os conceitos de Gleason, nos quais as comunidades sucessionais constituem "assembléias não-coesivas" e "o fenômeno da vegetação depende completamente do fenômeno sobre o indivíduo", sumarizam a escola de pensamento denominada "individualística". Algumas interpretações mais recentes e o próprio debate científico demonstram que a escala de observação pode ter grande influência na adoção de uma ou outra escola de pensamento (Shrader-Frechette \& McCoy, 1993). Nesta última, representada por Gleason, em parte transparecem idéias reducionistas, uma vez que a escala de observação tem como foco as partes do ecossistema ou da comunidade, sem enfatizar possíveis interrelações.

A despeito das distintas maneiras de observar as comunidades eecossistemas, assim como das possibilidades de variar os focos de observação, atualmente há indícios de que os processos sucessionais são modificados com a fragmentação e criação de bordas artificiais. Assim sendo, a correta interpretação dos fenômenos relacionados à fragmentação e aos efeitos de borda exige que sejam utilizados conceitos acerca da sucessão ecológica. Muito embora tais conhecimentos não sejam totalmente conclusivos, já fornecem bons indícios para fundamentar algumas interpretações.

Com isto, adotar uma perspectiva simplista para a classificação das espécies em grupos funcionais em resposta a distúrbios, por exemplo, aparentemente tem sido útil. Historicamente, um dos primeiros autores a utilizar e sumarizar tais idéias foi Budowski (1965). Mais recentemente, dentre os inúmeros seguidores de tal perspectiva, podem ser destacados Swaine \& Whitmore (1988), que ao incorporar novas informações aparentemente fortalecem os argumentos. Contudo, a ênfase nos grupos funcionais extremos (tolerante ou intolerante à sombra) continua, o que em certo aspecto perpetua o desconhecimento ao direcionar a interpretação. Em alguns trabalhos a confirmação de padrões é, em certo aspecto, involuntariamente tendenciosa. Entretanto, já existem indícios de que há um vasto gradiente entre estes extremos muitas vezes não observados devido à ênfase em aspectos como germinação, estabelecimento e sobrevivência de propágulos (Whitmore 1996). Além disto, a resposta exibida por uma espécie individualmente pode depender de uma complexa rede de inter-relações bióticas (Harper 1977). Portanto, a classificação precisa das espécies em grupos funcionais em resposta a distúrbios depende, em muitos casos, de um avanço expressivo no conhecimento acerca destas e dos processos e padrões relacionados a sua manutenção no respectivo habitat. Nesta perspectiva ainda que promissora a interpretação da sucessão ecológica a partir dos modelos de facilitação, tolerância e competição (vide McCook 1994), sua interpretação com relação aos limites artificiais é pouco elucidativa. Além disto, a visão estritamente mecanicista dos processos e padrões biológicos tem incorporado o fato de que eventos estocásticos podem ser mais 
freqüentes do que o esperado. Desta forma, o sucesso no estabelecimento de determinadas espécies vegetais não dependeria apenas de suas características selecionadas evolutivamente, mas também do acaso (Brokaw \& Busing 2000).

\section{OS EFEITOS DE BORDA}

Conforme já mencionado, atualmente, devido ao crescente desmatamento, há um grande aumento de limites artificiais (bordas), nos quais os ecossistemas e comunidades estão aparentemente sujeitos a efeitos de borda. Esta completa transformação da paisagem gera um cenário onde fragmentos relativamente pequenos e grandes compõem um arquipélago de ilhas de vegetação arbórea inseridos em uma matriz em geral graminóide ou arbustiva (Harris 1984; Laurance \& Bierregaard 1997). Tal perspectiva motivou inicialmente a utilização de idéias acerca da Biogeografia de Ilhas (e.g. MacArthur \& Wilson 2001) que pareciam adequadas para prever parte das conseqüências da fragmentação sobre os fragmentos remanescentes (e.g. Harris, 1984). Entretanto, recentemente notou-se que os fragmentos não representam necessariamente ilhas, e que as matrizes não são exatamente "mares inóspitos" (e.g. Laurance et al., 2002), sobretudo para determinados animais (Gascon et al. 1999). Com isto, determinadas previsões acerca da evolução das comunidades em fragmentos falharam ao utilizar a Biogeografia de Ilhas como modelo. Isto em parte levou a uma mudança de foco que atualmente enfatiza, por exemplo, a importância dos fenômenos que ocorrem nas bordas e nas florestas em regeneração próximas aos fragmentos (Pimm 1998; Mesquita et al. 1999). Portanto, nas tentativas de interpretar a fragmentação florestal, compreender como as bordas, que são cada vez mais freqüentes, se transformam no tempo tem sido um objetivo recorrente.

Uma borda recém criada, em termos teóricos, pode ser estruturalmente homogênea ou muito semelhante ao interior florestal. Ao longo do tempo, irão ocorrer outras transformações, relacionadas em grande parte aos efeitos de borda, que podem resultar em uma comunidade mais heterogênea neste limite. Porém, mesmo interiores florestais podem ser relativamente heterogêneos, ainda que mais homogêneos quando comparados a limites artificiais.

Em relação às transformações experimentadas pela borda, Matlack (1994) sumariza parte das idéias vigentes e identifica 3 fases: (1) formação, onde pode haver uma forte resposta da vegetação à criação do limite abrupto exibindo, inclusive, alta mortalidade; (2) reorganização dos gradientes físicos, onde pode ser desenvolvida uma camada de biomassa lateral que reduz a ação direta do vento e da insolação; e (3) expansão da área da borda, onde há efetivamente o estabelecimento de uma zona tampão biótica ou expansão da comunidade que passa a ter novos limites. A perspectiva utilizada por este autor, e compartilhada por outros, aparentemente é uma clara analogia à dinâmica de clareiras (e.g. Brokaw 1985) com respaldo no corpo teórico acerca da sucessão ecológica. Tais idéias são confirmadas por outros estudos que verificaram, por exemplo, maior mortalidade de árvores ao ser criado o limite (Lovejoy et al. 1986; Laurance et al. 1997; Kapos et al. 1997). Porém, considerando que vegetais são organismos modulares (Küppers 1994), a maior mortalidade poderia estar relacionada à falta de aclimatação de suas estruturas somáticas às novas condições. Por outro lado, algumas respostas do componente biótico, tais como a proliferação de lianas (e.g. Putz 1984) e bambus (e.g. Tabanez \& Viana 2000), previstas na fase (3), podem implementar um efeito sinérgico com o vento (Laurance et al. 2001), resultando na queda de grandes árvores. Em termos teóricos, a exclusão de indivíduos que dominam a paisagem, os quais possivelmente monopolizam grande parte dos recursos disponíveis, gera a oportunidade para o crescimento de outros indivíduos (e.g. juvenis ou propágulos). Neste sentido, nota-se que algumas bordas exibem maior densidade quando comparadas a interiores preservados (Laurance \& Bierregaard 1997; Oliveira-Filho 
et al. 1997; Laurance et al. 2002). Tal fato pode ser atribuído ao surgimento das oportunidades supracitadas, bem como à entrada de espécies associadas a estágios sucessionais menos avançados. Neste cenário, agora com espécies de crescimento rápido que teoricamente utilizam materiais metabolicamente menos custosos (Bazzaz 1991) podendo gerar estruturas somáticas mais frágeis, a comunidade seria mais dinâmica exibindo inclusive maior mortalidade (e.g. Oliveira-Filho et al. 1997). Ou seja, no caminho da estabilização dos fluxos de energia, a estrutura do ecossistema seria mais heterogênea e efêmera. Em estágios sucessionais mais avançados, teoricamente mais favoráveis à manutenção de grande biomassa e complexidade, haveria maior homogeneidade e durabilidade (vide Begon et al. 1995).

Deste modo, as transformações observadas com a criação de limites artificiais são, em geral, evidentes (Williams-Linera 1990; Laurance \& Yensen 1991; Camargo \& Kapos 1995; Laurance \& Bierregaard 1997; Tabanez et al. 1997; Cadenasso et al. 1997). Grande parte do problema reside então na forma de interpretar este cenário. Resgatando noções básicas em ecologia (vide Begon et al. 1995), alguns autores dividem os efeitos de borda em três tipos: (1) abióticos; (2) bióticos diretos e (3) bióticos indiretos (e.g. Murcia 1995). Assim sendo, as modificações no meio físico, que incluem aumento na insolação, velocidade do vento e queda na umidade relativa próximas às bordas, seriam propriamente exemplos de efeitos abióticos. Relacionados a estes, teoricamente, pode haver modificações diretas no componente biótico tais como aumento na densidade e entrada de espécies pioneiras. Os efeitos bióticos indiretos em geral são associados a processos tais como predação, herbivoria e polinização, que resultam de interações entre espécies. Estas perspectivas aparentemente são influenciadas pela idéia de compartimentalização em parte subjacente à teoria dos sistemas, na qual sistemas complexos apresentariam sub-unidades com menor complexidade (vide Margalef 1974).
Novamente em referência a evolução conceitual já citada, nota-se que as interações e os sinergismos entre os compartimentos podem ser extremamente complexos. Portanto, as subdivisões propostas podem ser artifícios didaticamente adequados à interpretação dos fenômenos relacionados à fragmentação florestal e efeitos de borda. Além disto, aparentemente há grande dificuldade em identificar precisamente fatores causais. Isto é particularmente percebido quando, mesmo ao identificar efeitos bióticos, a causa destes é associada a modificações abióticas (e.g. Murcia 1995).

Com isto, ainda é muito difícil determinar o conjunto de eventos no tempo que irá direcionar a trajetória dos processos ecológicos em bordas. Além disto, mesmo observando alguns padrões gerais pode haver distintos fatores causais. Grande parte dos estudos apenas identifica os processos e, conseqüentemente, os fatores direcionadores permanecem relativamente obscuros dificultando a detecção de padrões consistentes. Tal fato sugere que nas interpretações há uma mistura de fatores que são pouco elucidativos em relação aos processos e padrões intrínsecos à vegetação próxima a limites artificiais. Ainda que as informações sejam suficientes para indicar a existência de efeitos de borda, as consequiências destes em longo prazo também permanecem desconhecidas.

Evidentemente sob uma perspectiva intervencionista, onde a borda é um limite permeável sujeito a impactos advindos da matriz antrópica, a única conclusão é de que a degradação será crescente. Ainda mais se consideradas as escalas e intensidades de tais impactos. Por outro lado, em um cenário otimista, a interrupção dos impactos externos poderia permitir uma recuperação estrutural e funcional dos ecossistemas (e.g. Matlack 1994). Neste caso, a porosidade (permeabilidade) da borda poderia implementar um aumento na conectividade entre fragmentos ao invés de facilitar a entrada de impactos externos. Os trabalhos em áreas de regeneração (capoeiras) demonstram que a vegetação, 
dependend o das condições locais, pode de fato recuperar-se (e.g. Mesquita et al. 1999, 2001) re-estabelecendo assim inúmeras interações ecológicas do ecossistema (Gascon et al. 1999). Mesmo assim, é preciso considerar que uma redução expressiva na área (e.g. extinção de habitat) irá criar uma nova situação na qual certamente a coexistência de um grande número de espécies será improvável.

Considerando novamente a crescente degradação e os impactos ocasionados às bordas florestais, é esperado que o aspecto relativamente homogêneo exibido pelo ecossistema ou comunidade no momento da criação da borda, seja progressivamente tornado mais heterogêneo, podendo chegar a uma homogeneidade tardia. Esta última seria observada em locais onde os impactos advindos da matriz fossem recorrentes e intensos. Neste caso, a homogeneidade tardia seria caracterizada por processos e padrões típicos de ambiente de borda, nos quais também poderia haver um predomínio de espécies associadas a estágios sucessionais iniciais. Por outro lado, uma matriz menos agressiva poderia determinar impactos em menor escala e heterogeneamente distribuídos ao longo da borda. Desta forma, não haveria a referida homogeneidade tardia, mas sim um cenário altamente heterogêneo.

Neste ponto a idéia de ecótone, já mencionada anteriormente, permite uma interpretação bastante conveniente, onde fenômenos de retração e expansão podem ser explorados. Desta forma, praticamente todos os estudos sugerem que uma vez interrompidos os processos de degradação advindos da matriz há grande chance de que o remanescente se recupere ou se expanda.

Ainda em relação aos efeitos de borda, intuitivamente, é esperado que ocorram variações (em geral diminuição) na intensidade destes na medida em que aumentam as distâncias da borda (e.g. Kapos et al. 1997). Com isto, tornou-se freqüente descrever tais efeitos utilizando funções matemáticas relativas aos padrões observados e dentre estes, variando linearmente em função à distância da borda, identificam-se padrões "monotônicos" (e.g. Williams-Linera 1990). Por outro lado, padrões que oscilam em direção ao interior (bimodais ou em onda) são descritos utilizando modelos "não-monotônicos" (e.g. Franco \& Harper 1988). Nas perspectivas mencionadas transparecem idéias de que quanto maiores os fragmentos, maiores são as chances de que porções interiores destes estejam protegidas dos efeitos de borda (vide Laurance \& Yensen 1991; “The core area model.”). Este é um cenário relativamente simplista, mas que oculta uma grande complexidade, principalmente quando se considera a utilização de vários parâmetros simultaneamente. Além disto, mesmo fatores considerados simples e diretos quanto à medição (e.g. luz, vento), quando associados às respostas exibidas pelo componente biótico podem assumir complexas nuances. Estas, em parte, devido à inerente complexidade e heterogeneidade (vide MacIntosh 1991) dos sistemas biológicos.

\section{Conchusões}

Com a fragmentação florestalé inevitável a criação de bordas artificiais que podem implementar transformações aos sistemas biológicos ocasionadas em grande parte por efeitos de borda. O conhecimento sobre tais efeitos embora seja relativamente extenso ainda é pouco conclusivo devido principalmente ao desconhecimento dos processos e padrões naturais dos ecossistemas, falta de delimitação precisa dos fatores causais e deficiências nos conceitos e teorias utilizados. Por outro lado, considerando a natureza dinâmica dos ecossistemas, os fenômenos de expansão e retração e a tendência natural dos sistemas biológicos à evolução, supõe-se que as florestas também devem exibir respostas evolutivas em diversos níveis para lidar com este novo cenário. Neste aspecto, bordas florestais submetidas a matrizes extremamente impactantes (e.g. campos agrícolas) tenderiam a exibir um aspecto estruturalmente homogêneo determinado fortemente pelas transformações impostas pela matriz. Entretanto, matrizes menos impactantes 
poderiam favorecer a recuperação dos processos e padrões naturais dos ecossistemas exibindo nos locais onde há manutenção do limite abrupto um aspecto estruturalmente mais heterogêneo quando comparado a locais bem preservados. Os aspectos teóricos aqui abordados suportam tais argumentos, mas ainda assim permanecem incompletos e apontam para a necessidade de avançar no conhecimento sobre florestas tropicais, em especial considerando este cenário de intensa degradação e fragmentação. Por outro lado se a proliferação de bordas florestais não contribuir decisivamente para a perda de habitat e se os impactos aos remanescentes florestais forem minimizados é provável que os efeitos de borda não conduzam a uma crescente destruição.

\section{Agradecimentos}

Agradecemos especialmente ao Dr. Fábio Rúbio Scarano pelas suas valiosas sugestões nas versões iniciais deste trabalho. A João Marcelo de Alvarenga Braga, Mariana de Andrade Iguatemy, Rodolfo C. Real de Abreu, Pablo Viany Prieto e Bruno R. Silva (revisor do abstract) pelas sugestões e a dois assessores anônimos pela análise do manuscrito.

\section{REFERÊNCIAS BibLIOGRÁficas}

Ballaré, C. L. 1994. Light gaps, sensing the light opportunities in highly dynamic canopy environments. In: Caldwell, M. M. \& Pearcy, R. W. (eds.). Exploitation of environmental heterogeneity by plants. Academic Press, London, p. 73-110.

Bazzaz, F. A. 1991. Regeneration of tropical forests: physiological responses of pioneer and secondary species. In: Gómez-Pompa, A.; Whitmore, T. C. \& Hadley, M. (eds.). Rain Forest Regeneration and Management. UNESCO/Parthenon, Paris/Carnforth. Pp. 91-118.

Begon, M.; Harper, J. L. \& Townsend, C. R. 1995. Ecology: individuals, populations and communities. $2^{\text {nd }}$ ed. Blackwell Science, Cambridge. 945p.
Benitez-Malvido, J. 1998. Impact of forest fragmentation on seedling abundance in a tropical rain forest. Conservation Biology 12: 380-389.

Bierregard, R. O.; Lovejoy, T. E.; Kapos, V., Santos, A. A., \& Hutchings, R. W. 1992. The biological dynamics of tropical rainforest fragments. BioScience 42: 859-866.

Brokaw, N. V. L. 1982. The definition of treefall gap and its effect on measures of forest dynamics. Biotropica 14: 158-160. 1985. Gap-phase regeneration in a tropical forest. Ecology 66: 682-687.

Brokaw, N. \& Busing, R. T. 2000. Niche versus chance and tree diversity in forest gaps. Trends in Ecology and Evolution 15(5): 183-188.

Budowski, G. 1965. Distribution of tropical American rain forest species in the light of successional processes. Turrialba 15: 40-42.

Cadenasso, M. L.; Traynor, M. M. \& Pickett, S. T. A. 1997. Functional location of forest edges: gradients of multiple physical factors. Canadian Journal of Forest Research 27: 774-782.

Camargo, J. L. \& Kapos V. 1995. Complex edge effects on soil moisture and microclimate in central Amazonian forest. Journal of Tropical Ecology 11: 205-221.

Cowles, H. C. 1926. The fundamentals of vegetation change. Ecology 7: 391-413.

Cochrane, M. A. 2001. Synergistic interactions between habitat fragmentation and fire in evergreen tropical forests. Conservation Biology 15(6): 1515-1521.

\& Schulze D. 1999. Fire as recurrent event in tropical forests of the eastern Amazon: effects on forest structure, biomass and species composition. Biotropica 31: 2-16.

Cox, C. B. \& Moore, P. D. 1994. Biogeography: an ecological and evolutionary approach. Blackwell Scientific Publications. Oxford. 326p.

Dale, V. H. \& Pearson, S. M. 1997. Quantifying habitat fragmentation due to land use change 
in Amazonia. In: Laurance, W. F. \& Bierregaard, R. O. (eds.). Tropical Forest Remnants: ecology, management and conservation of fragmented communities. University of Chicago Press, Chicago \& London, p. 400-409.

Debinski, D. M. \& Holt, R. D. 2000. A survey and overview of habitat fragmentation experiments. Conservation Biology 14: 342-355.

Denslow, J. S. 1987. Tropical rainforest gaps and tree species diversity. Annual Review of Ecology and Systematics 18: 431-451.

Didham, R. K. \& Lawton, J. H. 1999. Edge structure determines the magnitude of changes in microclimate and vegetation structure in tropical forest fragments. Biotropica 31: 17-30.

Fahrig, L. 1997. Relative effects of habitat loss and fragmentation on population extinction. Journal of Wildlife Management 61(3): 603-610.

2003. Effects of habitat fragmentation on biodiversity. Annual Review of Ecology, Evolution and Systematics 34: 487-515.

Forman, R. T. T. 1995. Land Mosaics. The ecology of landscapes and regions, volumes I e II. Cambridge University Press, Cambridge: 321p. e 310p.

Franco, M. \& Harper, J.L. 1988. Competition and formation of spatial pattern in spatial gradients: an example using Kochia scoparia. Journal of Ecology 76: 959-974.

Gascon, C.; Lovejoy, T. E.; Bierregaard Jr., R. O.; Malcolm, J. R.; Stouffer, P. C.; Vasconcelos, H. L.; Laurance, W. F.; Zimmerman, B.; Tocher, M. \& Borges, S. 1999. Matrix habitat species richness in tropical forest remnants. Conservation Biology 91: 223-229.

Gleason, H. A. 1927. Further views on the succession concept. Ecology 8: 299-326.

Harper, J. L. 1977. Population Biology of Plants. Academic Press, London. 892p.

Harris, L. D. 1984. The fragmented forest. Chicago: University of Chicago Press. 211p.
Harrison, S. \& Bruna, E. M. 1999. Habitat fragmentation and large-scale conservation: what do we know for sure? Ecography 22: 225-232.

Holt, R. D.; Robinson, G. R \& Gaines, M. S. 1995. Vegetation dynamics in an experimentally fragmented landscape. Ecology 76: 1610-1624.

Holling, C. S. 1973. Resilience and stability of ecological systems. Annual Review of Ecology and Systematics 4: 1-23.

Hanski, I. 1985. Single-species dynamics may contribute to long-term rarity and commonness. Ecology 66: 335-343.

\& Gilpin, M. 1991. Metapopulation dynamics: brief history and conceptual domain. Biological Journal of the Linnean Society 42: 3-16.

Janzen, D. H. 1983. No park is an island: increase in interference from outside as park size decreases. Oikos 41: 402-410.

Kapos, V.; Wandelli, E.; Camargo, J. L. \& Ganade, G. 1997. Edge-related changes in environment and plant responses due to forest fragmentation in central Amazonia. In: Laurance, W. F. \& Bierregaard-Jr, R. O. (eds.). Tropical Forest Remnants: ecology, management, and conservation of fragmented communities. Chicago University Press. Chicago, p. 33-44.

Kareiva, P. \& Wennergren, U. 1995. Connecting landscape patterns to ecosystem and population processes. Nature 373: 299-302.

Küppers, M. 1994. Canopy gaps: competitive light interception and economic space filling-a matter of whole-plant allocation. In: Caldwell, M. M. \& Pearcy, R. W. (eds.). Exploitation of environmental heterogeneity by plants. Academic Press, London, p. 111-144.

Laurance, W. F. 2000. Do edge effects occur over large spatial scales? Trends in Ecology and Evolution 15: 134-135.

\& Bierregaard-Jr, R. O. (eds.). 1997. Tropical Forest Remnants: ecology, management and conservation of 
fragmented communities. Chicago: Chicago University Press. 616p.

; Didham, R.K. \& Power, M.E. 2001.

Ecological boundaries: a search for synthesis. Trendsin Ecology and Evolution 16(2): 70-71.

; Ferreira, L. V.; Rankin de Merona, J. M.; Laurance, S. G.; Hutchings, R. W. \& Lovejoy, T. E. 1998. Effects of fragmentation on recruitment patterns in Amazonian tree communities. Conservation Biology 12: 460-469.

; Laurance, S. G.; Ferreira, L. V.; Rankin de Merona, J. M.; Gascon, C. \& Lovejoy, T. E. 1997. Biomass collapse in Amazonian forest fragments. Science 278: 1117-1118.

; Lovejoy, T. E.; Vasconcelos, H. L.; Bruna, E. M.; Didham, R. K.; Stouffer, P. C.; Gascon, C.; Bierregaard, R. O.; Laurance, S. G. \& Sampaio, E. 2002. Ecosystem decay of Amazonian forest fragments: a 22-year investigation. Conservation Biology 16(3): 605-618.

\& Yensen, E. 1991. Predicting the impacts of edge effects in fragmented habitats. Biological Conservation 55: 77-92.

Lovejoy, T. E.; Bierregaard-Jr., R. O.; Rylands, A. B.; Malcon, J. R.; Quintela, C. E.; Harpe, L. H.; Brown, Jr.; K. S.; Powell, A. H.; Powell, G. V. N.; Schubart, H. O. R. \& Hays, M. B. 1986. Edge and other effects of isolation on Amazon forestfragments. In: Soulé, M. E. (ed.) Conservation Biology: The science of scarcity and diversity. Sinauer Associates. Sunderland. Pp. 257-285.

MacArthur, R. H. \& Wilson, E. O. 2001. The theory of island biogeography. Princeton University Press. Princeton. 224p.

MacIntosh, R. P. 1991. Concept and terminology of homogeneity and heterogeneity in ecology. In: Kolasa, J. \& Pickett, S. T. A. (eds.). Ecological heterogeneity. Springer-Verlag. New York: 25-46.

Margalef, R. 1974. Ecologia. Ediciones Omega. Barcelona. 951p.
Matlack, G. R. 1993. Microenvironmental variation within and among forest edge sites in the eastern United States. Biological Conservation 66: 185-194.

1994. Vegetation dynamics of the forest edge: trends in space and sucessional time. Journal of Ecology 82: 113-123.

McCook, L. J. 1994. Understanding ecological community sucession: Causal models and theories, a review. Vegetatio 110: 115-147.

Myers, N.; Mittermeier, R. A.; Mittermeier, C. G.; Fonseca, G. A. B. \& Kent, J. 2000. Biodiversity hotspots for conservation priorities. Nature 403: 853-858.

Mesquita, R. C. G.; Delamônica, P. \& Laurance, W. F. 1999. Effect of surrounding vegetation on edge-related tree mortality in Amazonian forest fragments. Biological Conservation 91: 129-134.

; Ickes, K.; Ganade, G. \& Williamson, G. B. 2001. Alternative successional pathways in the Amazon basin. Journal of Ecology 89: 528-537.

Morellato, L. P. C. \& Haddad, C. F. B. 2000. Introduction: the Brazilian Atlantic forest. Biotropica 32(4b): 786-792.

Moses, C. 1989. A geochemical perspective on the causes and periodicity of mass extinctions. Ecology 70(4): 812-823.

Murcia, C. 1995. Edge effects in fragmented forests: implications for conservation. Trends in Ecology and Evolution 10:58-62.

Odum, E. P. 1964. The new ecology. BioScience 14: 14-16.

Oliveira-Filho, A. T.; Mello, J. M. \& Scolforo, J. R. S. 1997. Effects of past disturbance and edges on tree community structure and dynamics within a fragment of tropical semideciduous forest on south-eastern Brazil over a five-year period (1987-1992). Plant Ecology 131: 45-66.

Patterson, C. \& Smith, A. B. 1989. Periodicity in extinction: the role of systematics. Ecology 70(4): 802-811. 
Pickett, S. T. A.; Cadenasso, M. L. \& Jones, C. G. 2000. Generation of heterogeneity by organisms: creation, maintenance and transformation. In: Hutchings, M.J.; John, E. A. \& Stewart, A. J. A. (eds.). The ecological consequences of environmental heterogeneity. Blackwell Science. London. Pp. 33-52.

Pimm, S. L. 1991. The Balance of Nature? Ecological issues in the conservation of species and communities. University of Chicago Press. Chicago. 554p. 1998. The forest fragment classic. Nature 393: 23-24.

Putz, F. E. 1984. The natural history of lianas on Barro Colorado island, Panama. Ecology 65: 1713-1724.

Saunders, D. A.; Hobbs, R. J. \& Margules, C. R. 1991. Biological consequences of ecosystem fragmentation: a review. Conservation Biology 5: 18-32.

Scarano, F.R.; Duarte, H. M.; Ribeiro, K. T.; Rodrigues, P. J. F. P.; Barcellos, E. M. B.; Franco, A. C.; Brulfert, J.; Deléens, E. \& Lüttge, U. 2001. Four sites with contrasting environmental stress in southeastern Brazil: relations of species, life form diversity, and geographical distribution to ecophysiological parameters. Botanical Journal of the Linnean Society 136: 345-364.

Shrader-Frechette, K. S. \& McCoy, E. D. 1993. Method in ecology: strategies for conservation. Cambridge University Press. $328 \mathrm{p}$.

Sih, A.; Jonsson, B. G., \& Luikart, G. 2000. Habitat loss: ecological, evolutionary and genetic consequences. Trends in Ecology and Evolution 15: 132-134.

Swaine, M. D \& Whitmore, T. C. 1988. On the definition of ecological species groups in tropical rain forests. Vegetatio 75: 81-6.

Tabanez, A. A. J. \& Viana, V. M. 2000. Patch structure within Brazilian atlantic forest fragments and implications for conservation. Biotropica 32(4b): 925-933.

Tilman, D. 1999. Global environmental impacts of agricultural expansion: The need for sustainable and efficient practices. Proceedings of the National Academy of Sciences USA 96: 5995-6000.

Tilman, D.; May, R. M.; Lehman, C. L. \& Nowak, M. A. 1994. Habitat destruction and the extinction debt. Nature 370: 66.

Viana, V. M. 1995. Conservation of biological diversity in Neotropical forest fragments in intensively cultivated landscapes. In: Fonseca, G. S. M.; Schmink, M.; Pinto, C. P. S. \& Brito, F. (eds.) On common ground: Interdisciplinary approaches to biodiversity conservation and land use dynamics in the New World. Conservation International, Belo Horizonte, Brazil.

Weiner, J. 1988. Variation in performance of individuals in plant populations. In: Davy, A. J.; Hutchings, M. J. \& Watkinson, A. R. (eds.). Plant Population Ecology. Blackwell, Oxford, p. 59-81.

Whitmore, T. C. 1989. Canopy gaps and the two major groups of forest trees. Ecology 70: 536-538

1991. An introduction to tropical rain forests. Clarendon Press. Oxford. $226 \mathrm{p}$. 1996. A review of some aspects of tropical rain forest seedling ecology with suggestions for further enquiry. In: Swaine, M. D. (ed.). The ecology of tropical forest tree seedling. UNESCO/ Parthenon, Paris /Carnforth: 3-49.

Williams-Linera, G. 1990. Vegetation structure and environmental conditions of forest edges in Panama. Journal of Ecology 78: 356-373.

Watt, A. S. 1947. Pattern and process in the plant community. Journal of Ecology 35: 1-22.

Wiens, J. A. 1989. Spatial Scaling in Ecology. Functional Ecology 3: 385-397.

WCMC - World Conservation Monitoring Center. 1992. Global biodiversity: status of the Earths living resources. London, Chapman \& Hall. 585p. 
